\title{
EVALUACIÓN DE METABUSCADORES GRATUITOS ESPECIALIZADOS EN CIENCIAS DE LA SALUD
}

\author{
Sílvia Sastre-Suárez y Elena Pastor-Ramon
}
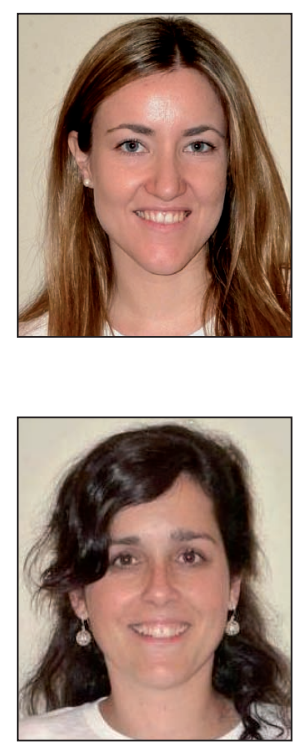

Resumen

Sílvia Sastre-Suárez es licenciada en documentación y diplomada en biblioteconomía y documentación por la Universitat de Barcelona. Responsable de la Unitat de Documentació Biomèdica de la Gerència d'Atenció Primària de Mallorca. Colabora con la Biblioteca Virtual de Ciències de la Salut de las Illes Balears. Como docente ha impartido sesiones bibliográficas en los Centros de Salud de Mallorca, y cursos de formación sobre documentación científica en ciencias de la salud.

Biblioteca Virtual de Ciències de la Salut de les Illes Balears Hospital Universitari Son Espases. Biblioteca (mòdul L +1) Ctra. Valldemossa, 79, Mallorca. 07120 Illes Balears, España ssastre@bibliosalut.com http://www.bibliosalut.com

Elena Pastor-Ramon, licenciada en documentación por la Universitat Oberta de Catalunya y diplomada en biblioteconomía y documentación por la Universidad de Salamanca, es documentalista de la Fundació Hospital Son Llàtzer, Palma de Mallorca. Colaboradora de la Unitat Técnica de la Biblioteca Virtual de Ciències de la Salut de les Illes Balears. Es docente de diferentes cursos sobre documentación en ciencias de la salud.

FBiblioteca Virtual de Ciències de la Salut de les Illes Balears Hospital Universitari Son Espases. Biblioteca (mòdul L +1) Ctra. Valldemossa, 79, Mallorca. 07120 Illes Balears, Españae pastor@bibliosalut.com

Continuamente aparecen nuevos sistemas de recuperación de la información (buscadores, metabuscadores, bases de datos, etc.), pero el trabajo diario de los profesionales de la documentación deja poco tiempo para su evaluación. Ello trae consigo que en numerosas ocasiones se utilicen e incluso recomienden algunos de los que no se conocen realmente sus funciones y la adecuación de su algoritmo de búsqueda. Por ello se realizó un análisis para conocer mejor las especificidades de los buscadores especializados en ciencias de la salud accesibles gratuitamente a través de internet, para cerciorarnos de su calidad y saber cuáles ofrecen mejores resultados.

\section{Palabras clave}

Recursos electrónicos, Evaluación de recursos, Metabuscadores, Sistemas avanzados de recuperación de la información.

\section{Title: Evaluation of free metasearch engines specialized in health sciences}

\section{Abstract}

Although new information retrieval systems (search engines, metasearch engines, databases, etc.) continually appear, the daily work of information professionals leaves little time to evaluate them. As a result, on numerous occasions a system is used, and even recommended, without knowledge of its functions and the adequacy of its search algorithm. An analysis was conducted to better understand the specifics of the search engines specialized in health sciences freely accessible via the internet, to assess for quality and determine which ones offer the best results.

\section{Keywords}

Electronic resources, Resource assessment, Evaluation, Metasearch, Advanced information retrieval.

Sastre-Suárez, Sílvia; Pastor-Ramon, Elena. "Evaluación de metabuscadores gratuitos especializados en ciencias de la salud". El profesional de la información, 2011, noviembre-diciembre, v. 20, n. 6, pp. 639-644.

http://dx.doi.org/10.3145/epi.2011.nov.06

\section{Introducción}

A los profesionales de la información nos interesa conocer en profundidad el funcionamiento y las características de los metabuscadores gratuitos online, tanto para nuestro

Artículo recibido el 29-04-11

Aceptación definitiva: 31-08-11 uso como para recomendar a los usuarios. Por ello, el presente estudio tiene como objetivo evaluar algunos de los metabuscadores gratuitos más destacados y especializados en ciencias de la salud, a nivel nacional e internacional, para 


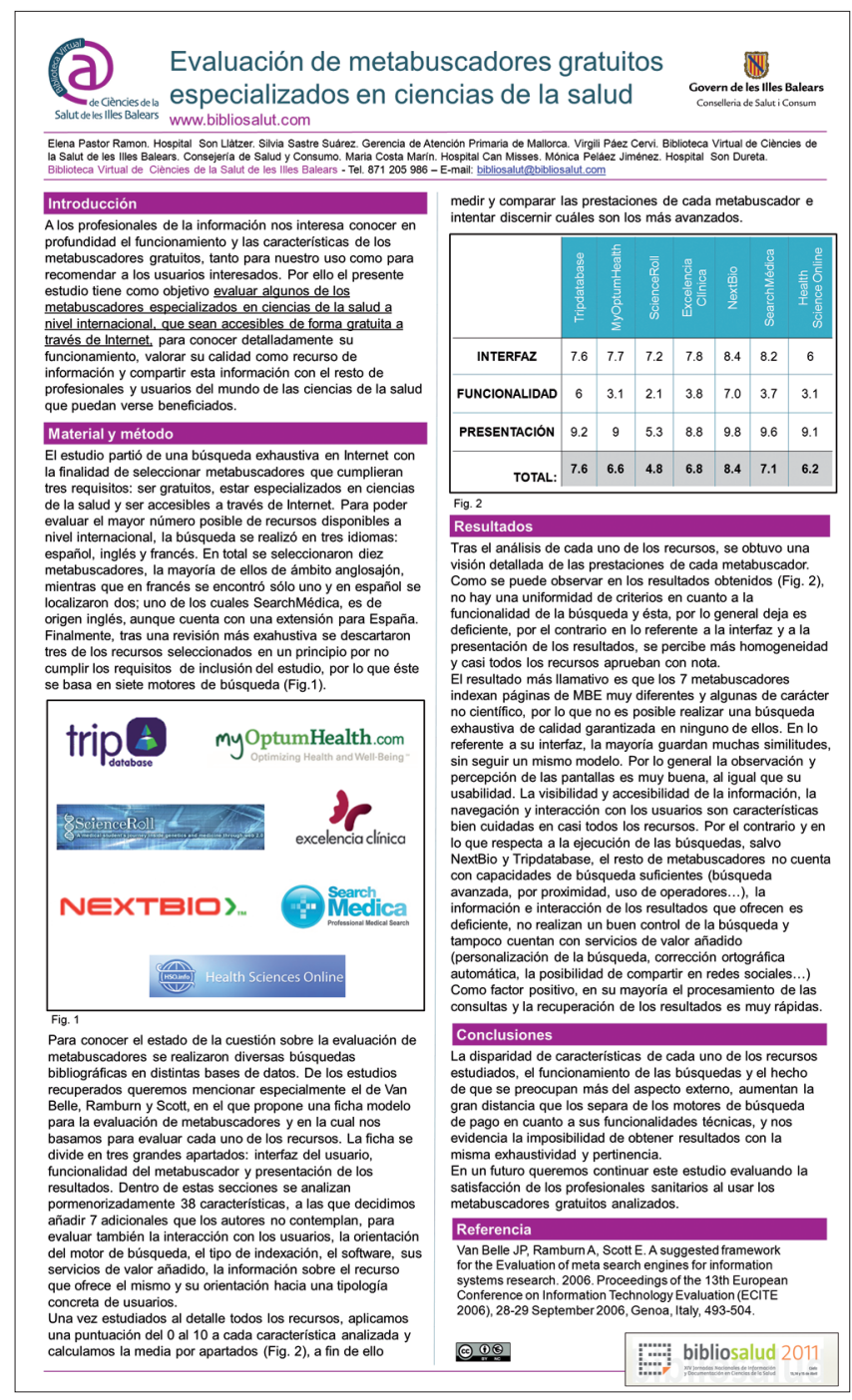

Figura 1. http://eprints.rclis.org/handle/10760/15985

conocer detalladamente su funcionamiento, valorar su calidad como recurso de información y compartir esta información con el resto de profesionales y usuarios del mundo de las ciencias de la salud. Este estudio se basa en un póster (Pastor-Ramon; Sastre-Suárez; Páez et al., 2011) (figura 1) presentado en las XIV Jornadas nacionales de información y documentación en ciencias de la salud, que posteriormente ha sido revisado y ampliado dado el interés de los autores de este artículo por profundizar en el tema y extraer más resultados de utilidad para nuestra comunidad científica.

El trabajo diario de los profesionales de la documentación deja poco tiempo para la evaluación de recursos

\section{Selección y análisis previo de los metabuscadores}

El estudio partió de una búsqueda exhaustiva con la finalidad de seleccionar los metabuscadores que cumplieran tres requisitos: ser gratuitos, estar especializados en ciencias de la salud y ser accesibles a través de internet.
Para poder evaluar el mayor número posible de recursos disponibles a nivel internacional, la búsqueda se realizó en tres idiomas: español, inglés y francés. Tras ésta en total se seleccionaron 10 metabuscadores, la mayoría de ellos de ámbito anglosajón, mientras que en francés sólo se encontró 1 y en español se localizaron 2, uno de los cuales Search Medica es de origen inglés, aunque cuenta con una extensión para España. Los seleccionados fueron: Excelencia Clínica, Next Bio, ScienceRoll, Scirus, Search Medica, Trip Database, My Optum Health, Google Health, Moteur de recherche médicale y Health Science Online.

Tras una última revisión se descartaron 3 de los recursos seleccionados en un principio, por no cumplir los requisitos de inclusión del estudio, por lo que finalmente éste se basa en 7 motores de búsqueda (figura 2). Los descartados fueron: Scirus, por ser generalista, no analiza recursos únicamente de ciencias de la salud, es multidisciplinar; Google Health, por tratarse de un proyecto de historia clínica electrónica universal y no de un metabuscador especializado; y Moteur de recherche médicale, por estar creado a partir de Google, que no garantiza una búsqueda exhaustiva sobre salud en toda la Web que sea de calidad.

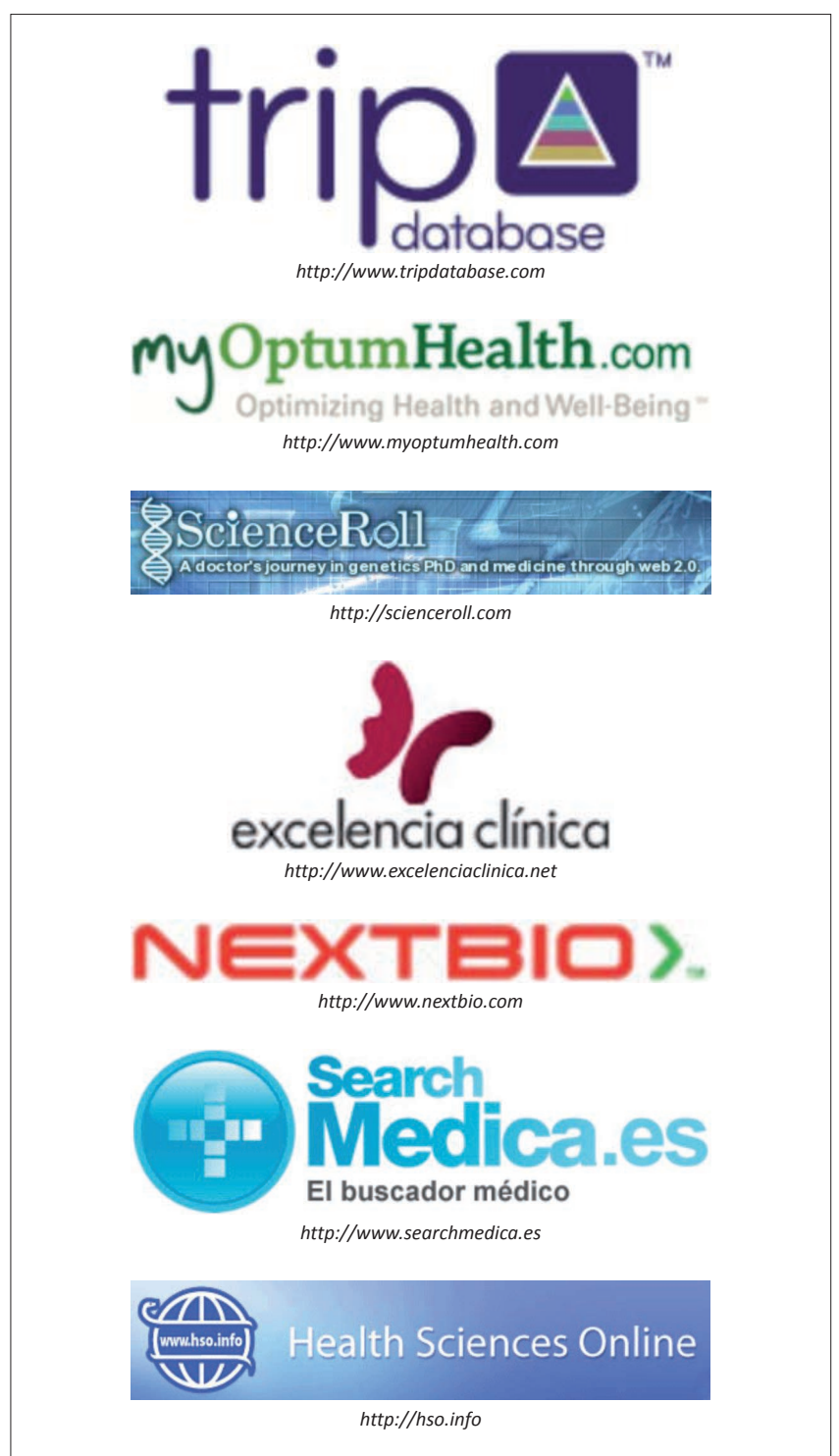

Figura 2. Metabuscadores analizados 


\section{Análisis de los metabuscadores}

Para conocer el estado de la cuestión sobre la evaluación de metabuscadores se realizaron diversas búsquedas bibliográficas en distintas bases de datos y repositorios de acceso abierto sobre biblioteconomía, documentación y ciencias de la salud. Nos llamaron especialmente la atención los estudios de Codina (2000) sobre la evaluación de recursos digitales, el libro de Jiménez y Ortiz-Repiso (2007) sobre evaluación de sedes web y el artículo de Guallar y Abadal (2009) sobre evaluación de hemerotecas de prensa digital. Del resto queremos mencionar especialmente el de Van BeIle; Ramburn y Scott (2006), en el que propone una ficha modelo para la evaluación de metabuscadores (tabla 1 ) y en la cual nos basamos para evaluar cada uno de ellos. La ficha se divide en tres grandes apartados:

1. Interfaz del usuario.

2. Funcionalidad del metabuscador.

3. Presentación de los resultados.

Además se analizaron otras características adicionales, que pueden verse en la tabla 2 .

Dentro de estas secciones se valoran 38 características, a las que decidimos añadir 7 adicionales que los autores citados no contemplan (destacadas con ${ }^{*}$ ), para evaluar también:

- opciones ofrecidas de interacción con los usuarios;

- orientación del motor de búsqueda hacia un tipo de información (formato) concreto;

- tipo de indexación automática o manual;

- software que hay detrás y lenguaje de programación utilizado;

- servicios de valor añadido que incluye: cuenta de correo electrónico, clipboard (portapapeles para copiar temporalmente), etc.;

- información ofrecida sobre el metabuscador tanto a nivel técnico como de usuario; y

- orientación hacia un tipo concreto de usuario.

Por otro lado fueron eliminados varios campos, como el de "filtro de material para adultos", ya que no era relevante conocer si disponía de dicha característica dada la especialidad en ciencias de la salud de los buscadores.

La ficha se divide en tres grandes apartados: interfaz del usuario, funcionalidad del metabuscador y presentación de los resultados

\section{Resultados}

\subsection{Extracción de los resultados}

Una vez analizadas al detalle todas las características de los recursos, se aplicó una puntuación del 0 al 10 a cada una para valorarlas y poder así calcular la media por apartados (tabla 3). Cabe puntualizar que cuando la característica no podía ser valorada en una escala del 0 al 10 sino que se trataba de tenerla o no tenerla, ésta fue puntuada con un 10 si el metabuscador contaba con la característica y con un 0 si no lo hacía.

\begin{tabular}{|c|c|}
\hline \multirow{10}{*}{ 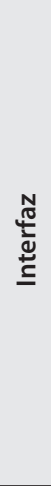 } & Observación y percepción \\
\hline & Atractivo y profesionalidad \\
\hline & Simplicidad \\
\hline & Consistencia \\
\hline & Usabilidad \\
\hline & Visibilidad de la información \\
\hline & Accesibilidad de la información \\
\hline & Navegación \\
\hline & Interacción con los usuarios* \\
\hline & Funcionalidad del metabuscador \\
\hline \multirow{10}{*}{ 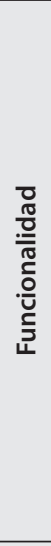 } & Capacidades de búsqueda \\
\hline & Búsqueda avanzada \\
\hline & Uso de operadores booleanos \\
\hline & Sugerencia de resultados \\
\hline & Frase exacta \\
\hline & Truncamientos \\
\hline & Diferentes opciones de búsqueda (imágenes, artículos, etc.) \\
\hline & $\begin{array}{l}\text { Orientación a la búsqueda de un tipo (formato) de información } \\
\text { concreto* }^{*}\end{array}$ \\
\hline & Búsqueda por lenguaje natural \\
\hline & $\begin{array}{l}\text { Sistema de indexación de los contenidos (automático o } \\
\text { manual)* }\end{array}$ \\
\hline \multirow{15}{*}{ 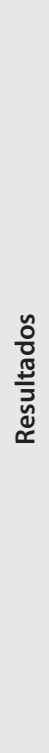 } & Presentación de los resultados \\
\hline & Descripción \\
\hline & Dirección electrónica del recurso \\
\hline & Opción de volver \\
\hline & Categorización de los resultados \\
\hline & Numeración de los resultados \\
\hline & Ordenación de los resultados por relevancia \\
\hline & $\begin{array}{l}\text { Fuentes/buscadores de donde obtiene los resultados el } \\
\text { metabuscador }\end{array}$ \\
\hline & Visualización de los resultados en una nueva ventana \\
\hline & Selección de los resultados según su idioma \\
\hline & Control de la búsqueda \\
\hline & Selección del criterio de obtención de resultados \\
\hline & Fecha \\
\hline & Limitación del número de resultados \\
\hline & Filtración de dominios no deseados \\
\hline
\end{tabular}

Tabla 1. Características básicas analizadas

Para valorar la funcionalidad de los recursos analizados, era necesario probar su funcionamiento por lo que se realizó la misma búsqueda en todos ellos. El tema escogido fue "Dieta alimenticia en mujeres embarazadas que presentan diabetes gestacional".

\subsection{Destacados}

Tras el análisis se obtuvo una visión detallada de las prestaciones de cada metabuscador. Como se puede observar en los resultados obtenidos (tabla 3 ), no hay una uniformidad de criterios en cuanto a la funcionalidad de la búsqueda, y ésta, por lo general es deficiente. Por el contrario en lo relativo a la interfaz y a la presentación de los resultados se percibe más homogeneidad y casi todos los recursos aprueban con nota. La mayoría cuida más la imagen externa que su 


\begin{tabular}{|l|}
\hline \multicolumn{1}{|c|}{ Supervisión del sistema } \\
\hline Procesamiento de las consultas (rapidez) \\
\hline Recuperación de los resultados (cantidad) \\
\hline $\begin{array}{l}\text { Visualización de las búsquedas relacionadas de otros usuarios (auto- } \\
\text { sugerencia al escribir en la casilla de búsqueda) }\end{array}$ \\
\hline Software que lo soporta, lenguaje html que utiliza, etc.* \\
\hline \multicolumn{1}{|c|}{ Practerísticas deseables } \\
\hline Ayuda/FAQ Cocisión \\
\hline Personalización de la barra de búsqueda \\
\hline Autocorrección ortográfica de las estrategias de búsqueda \\
\hline $\begin{array}{l}\text { Inclusión de servicios de valor añadido (cuenta de correo electrónico, } \\
\text { clipboard, etc.)* }\end{array}$ \\
\hline $\begin{array}{l}\text { “About us" información sobre el propio recurso para saber como } \\
\text { funciona* }\end{array}$ \\
\hline Presentación \\
\hline \multicolumn{1}{c|}{ Estabilidad } \\
\hline $\begin{array}{l}\text { Número de documentos relevantes recuperados x Número total de } \\
\text { documentos recuperados }\end{array}$ \\
\hline
\end{tabular}

Tabla 2. Características adicionales de los metabuscadores

funcionamiento, lo que lleva a que los resultados esperados al realizar la búsqueda no sean los deseados en la mayoría de casos.

El resultado más llamativo es que los 7 metabuscadores indexan páginas de medicina basada en la evidencia (MBE) muy diferentes y algunas de carácter no científico, por lo que no es posible garantizar una búsqueda adecuada y de calidad en ninguno de ellos.

\section{En la página principal todos tienen una casilla de búsqueda, respondiendo a un diseño claro y ordenado}

\subsection{Interfaz}

Las diferentes interfaces guardan muchas similitudes, sin seguir un mismo modelo. Por lo general la apariencia y la estructura son muy claras y la usabilidad buena. La visibilidad y accesibilidad de la información, la navegación e interacción con los usuarios son características bien cuidadas en casi todas. En la página principal tienen una casilla de búsqueda, respondiendo a un diseño claro y ordenado, que se ve reproducido en el resto de páginas secundarias, lo que confiere coherencia. Aun así algunas webs combinan este tipo de búsqueda mediante lenguaje libre con la búsqueda por materias de un listado o de guías en apartados diferentes, lo que puede desorientar al usuario. Pensamos que es mejor una única casilla de búsqueda desde la que se obtiene un listado de todos los resultados, sin necesidad de familiarizarse previamente con la arquitectura del recurso, o sin tener que ir abriendo y cerrando pantallas innecesariamente para llegar a la información que se desea.

Desde el punto de vista de la usabilidad hemos observado una gran disparidad en las funciones específicas que ofrecen los distintos metabuscadores, y en la manera de mostrar, acceder y navegar por los resultados. Ello es debido a que aunque la mayoría son recursos referenciales, también los hay a texto completo pero que antes hacen pasar por una ficha descriptiva. Bajo nuestro punto de vista éste tendría que ser un aspecto más cuidado por los metabuscadores: deberían hacer un esfuerzo por unificar todos los aspectos de usabilidad. Igualándose y haciéndose más intuitivos serían más cómodos para los usuarios, los cuales no necesitarían familiarizarse con ellos antes de usarlos. Creemos necesario un consenso en nuestra comunidad científica en la consecución de unos estándares a seguir por todos los sistemas avanzados de recuperación de la información que certifiquen su calidad como recurso de información.

\section{Es necesario un consenso para crear es- tándares de funcionamiento que sean adoptados por todos los metabuscadores}

Otro aspecto a destacar de las interfaces es que sólo una minoría incorpora prestaciones para que los usuarios puedan interactuar. En este punto vemos imprescindible que cuenten con herramientas de sindicación de contenidos (RSS) para facilitar la revisión de los temas. El no disponer de ellas hace que a la larga sean descartados al no permitir mantenerse actualizado de forma automática. Algunas de las plataformas más conocidas en ciencias de la salud, como PubMed, ya hace tiempo que cuentan con este tipo de herramientas. Los desarrolladores de los metabuscadores estudiados deberían hacer un ejercicio de benchmarking con los sistemas más avanzados y ponerse al día.

\begin{tabular}{|l|c|c|c|c|c|c|c|}
\hline & Trip database & $\begin{array}{c}\text { My Optum } \\
\text { Health }\end{array}$ & Science Roll & $\begin{array}{c}\text { Excelencia } \\
\text { Clínica }\end{array}$ & Next Bio & $\begin{array}{c}\text { Search Medica } \\
\text { Health Science } \\
\text { Online }\end{array}$ \\
\hline Interfaz & $\mathbf{7 , 6}$ & 7,7 & 7,2 & 7,8 & 8,4 & 8,2 & 6,0 \\
\hline Funcionalidad & 6,0 & 3,1 & 2,1 & 3,8 & 7,0 & 3,7 & 3,1 \\
\hline Presentación & 9,2 & 9,0 & 5,3 & 8,8 & 9,8 & 9,6 & 9,1 \\
\hline Total & $\mathbf{7 , 6}$ & $\mathbf{6 , 6}$ & $\mathbf{4 , 8}$ & $\mathbf{6 , 8}$ & $\mathbf{8 , 4}$ & $\mathbf{7 , 1}$ & $\mathbf{6 , 2}$ \\
\hline
\end{tabular}

Tabla 3. Resultado del análisis 


\subsection{Funcionalidad}

En lo que respecta a la ejecución de las búsquedas, salvo Next Bio y Trip Database, el resto de metabuscadores no cuenta con capacidades de búsqueda suficientes (búsqueda avanzada, por proximidad, uso de operadores...). En un principio todos los metabuscadores parecen ofrecer estos servicios, pero al ejecutar la búsqueda vemos que realmente no es así. No ofrecer búsqueda avanzada no supone una desventaja importante para los usuarios que quieren una vía de acceso a la información sencilla y rápida, pero es una lástima que no se pueda limitar por años, tipos de documento, instituciones de los que provienen, etc.

La mayoría de metabuscadores analizados realizan la búsqueda en lenguaje libre, ello tiene algunas ventajas, como pueden ser la rapidez y facilidad para realizar la búsqueda, y algunos inconvenientes como el no poder utilizar tesauro o la limitación por campos.

La mayoría de los metabuscadores ejecutan la búsqueda únicamente en un idioma, el inglés, que es el de la mayor parte de la producción científica sobre ciencias de la salud. Esto es un handicap, ya que se pierden muchos resultados pertinentes escritos en otras lenguas. En este sentido destaca el metabuscador Excelencia Clínica, del Ministerio de Sanidad de España, que ha tenido en cuenta este punto incluyendo resultados en inglés cuando el usuario realiza la búsqueda en castellano.

En definitiva consideramos la funcionalidad y la exhaustividad de los metabuscadores las características más importantes para garantizar su calidad, pero lamentablemente varios de los analizados en el presente estudio no realizan realmente una búsqueda completa, tal y como demuestran los resultados obtenidos. Se observa una gran disparidad de resultados, no sólo posible por el hecho comentado anteriormente de que cada uno indexa recursos diferentes, sino también porque sus algoritmos de búsqueda son muy diferentes.

\subsection{Presentación de los resultados}

En lo que concierne a la precisión de los resultados hemos analizado el número de documentos pertinentes recuperados dentro del número total de documentos. En la mayoría de búsquedas sólo son pertinentes los primeros resultados. Algunos investigadores mencionan que sólo algunos usuarios saben juzgar la relevancia de los documentos en búsquedas con muchos resultados (Bar-Ilan; Mat-Hassan; Levene, 2006), aunque no podemos saber si son o no relevantes para un usuario determinado, pues como comentan Marcos y González-Caro (2010) ello depende de si el usuario es informacional o navegacional.

También estudiamos la estabilidad de los resultados. Es decir si haciendo la misma búsqueda en momentos diferentes o días diferentes daba los mismos resultados. Y observamos que en la mayoría de recursos era así.

Todos los metabuscadores, excepto Science Roll, son muy rápidos. El tiempo total entre realizar la búsqueda y recuperar los resultados es de muy pocos segundos.

Igualmente todos cumplen el requisito de compatibilidad con diferentes navegadores: Internet Explorer, Mozilla FireFox y Chrome.
Por otro lado no disponen de mecanismos de interacción con los resultados ni servicios de personalización, corrección ortográfica automática, posibilidad de compartir en redes sociales, etc. Deberían replantearse a qué tipo de usuario está destinada la información que ofrecen y adaptarse a sus necesidades. Algunos como Search Medica, Trip Database, My Optum Health y Health Sciences Online están destinados solamente a los profesionales de las ciencias de la salud (médicos, enfermeros, psicólogos, etc.). Excelencia Clínica también se dirige a los pacientes y, Next Bio y Science Roll se orientan a disciplinas que se relacionan con la salud, como por ejemplo la genética o la biología, entre otras materias.

\section{Conclusión}

La disparidad de características de cada uno de los recursos estudiados, el mal funcionamiento de las búsquedas y el preocuparse más del aspecto externo que de su funcionalidad, hace que los metabuscadores gratuitos estén a una gran distancia de los de pago. Es evidente la imposibilidad de obtener resultados con la misma exhaustividad y pertinencia. Estos recursos deberían hacer un mayor esfuerzo para mejorar y uniformizar sus características esenciales.

\section{Recomendaciones}

Desde la visión que nos han ofrecido a día de hoy estos 7 metabuscadores, recomendamos a sus usuarios potenciales que no se queden con la interrogación en uno solo, si lo que quieren es una consulta exhaustiva de los materiales que puedan encontrarse sobre un tema determinado en toda la Web.

Por otro lado, aunque el primer paso que den sea la elección de uno de estos recursos gratuitos, les animamos a no dejar de usar los medios que ponen a su disposición las bibliotecas de ciencias de la salud para obtener más precisión y calidad.

No debe olvidarse realizar las búsquedas prioritariamente en inglés y después en otros idiomas secundarios en el mundo de la investigación.

Recomendamos a los desarrolladores de los metabuscadores estudiados que no se centren únicamente en el aspecto externo. Es una lástima que no aprovechen los muchos avances técnicos existentes que les permitirían dar más valor a sus plataformas, tales como la conexión con redes sociales y la búsqueda avanzada (por campos). Igualmente es importante que el metabuscador categorice los resultados, tal como hace Trip Database, pues de esta forma el usuario puede ir rápidamente a los que le interesan. Y finalmente les sugerimos que aporten más información sobre las fuentes de las que extraen la información, ya que conocer cuáles indexan es esencial para garantizar la fiabilidad de la información.

\section{Bibliografía}

Arbildi-Larreina, Iñigo. "Posicionamiento en buscadores: una metodología práctica de optimización de sitios web". El profesional de la información, 2005, marzo-abril, v. 14, n. 2, pp. 108-124. 
http://www.elprofesionaldelainformacion.com/contenidos/ 2005/marzo/3.pdf

Bar-Ilan, Judit; Mat-Hassan, Mazlita; Levene, Mark. Methods for comparing rankings of search engine results. Computer networks, 2006, v. 50, pp. 1448-1463.

http://dx.doi.org/10.1016\%2Fj.comnet.2005.10.020

Codina, Lluís. "Evaluación de recursos digitales en línea: conceptos, indicadores y métodos". Revista española de documentación científica, 2000, v. 23, n. 1, p. 9-44.

http://redc.revistas.csic.es/index.php/redc/article/viewArti cle/315

Codina, Lluís. Metodología de análisis y evaluación de recursos digitales en línea. Barcelona: UPF. Área de Biblioteconomía y Documentación. Dep. Periodismo y Comunicación Audiovisual, 2006, 56 pp.

http://www.lluiscodina.com/metodos/metodos2006.doc

García-De-León, Alicia; Caldera-Serrano, Jorge. "Una herramienta de evaluación de buscadores internos para la valoración de medios de prensa escrita en la Red". Cuadernos de documentación multimedia, 2007, n. 18.

http://multidoc.rediris.es/cdm/include/getdoc.php?id= 252\& article $=47$

Guallar, Javier; Abadal, Ernest. Evaluación de hemerotecas de prensa digital: indicadores y ejemplos de buenas prácticas. El profesional de la información, 2009, v. 18, n. 3, pp. 255-269. http://eprints.rclis.org/handle/10760/13048 http://dx.doi.org/10.3145/epi.2009.may.02

Jiménez, Marina; Ortiz-Repiso, Virginia. Evaluación y calidad de sedes web. Gijón: Trea, 2007.

Mahoney, William; Hospodka, Peter; Sousan, William L.; Nickell, Ryan; Zhu, Qiuming. A coherent measurement of web search relevance. Technical report n. Cst-2008-2.

http://cs.unomaha.edu/trpt/2008/cst-2008-2.pdf

Marcos, Mari-Carmen; González-Caro, Cristina. "Comportamiento de los usuarios en la página de resultados de los buscadores. Un estudio basado en eye tracking". El profesional de la información, 2010, julio-agosto, v. 19, n. 4, pp. 348-358.

http://dx.doi.org/10.3145/epi.2010.jul.03

Pastor-Ramon, Elena; Sastre-Suárez, Sílvia; Páez, Virgili, et al. Evaluación de metabuscadores gratuitos especializados en ciencias de la salud, 2011. Poster. En: XIV Jornadas nacionales de información y documentación en ciencias de la salud, Cádiz, 13-15 de abril de 2011.

http://eprints.rclis.org/handle/10760/15985

Van Belle, Jean-Paul; Ramburn, Anjali; Scott, Elsje. "A suggested framework for the evaluation of meta search engines for information systems research". In: Proceedings of the $13^{\text {th }}$ European conf on information technology evaIuation (Ecite 2006), 28-29 Sept 2006, Genova, Italia, pp. 493-504.

\section{SerialsSolutions}

\section{A ProQuest Company}

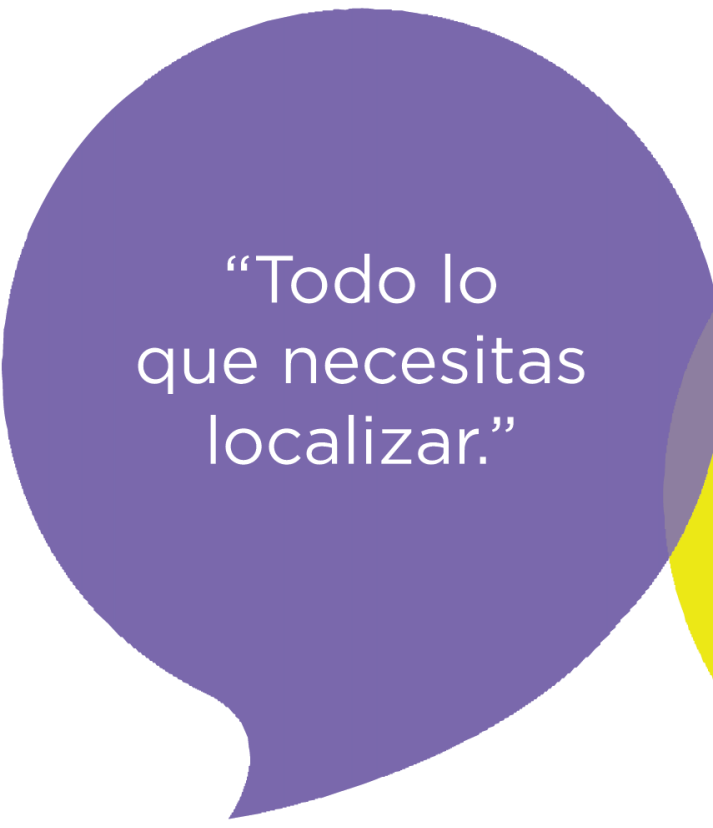

Descubre Summon $^{\text {TM }}$ para tu biblioteca
"Fácil de usar."
"Centrémonos en la información."
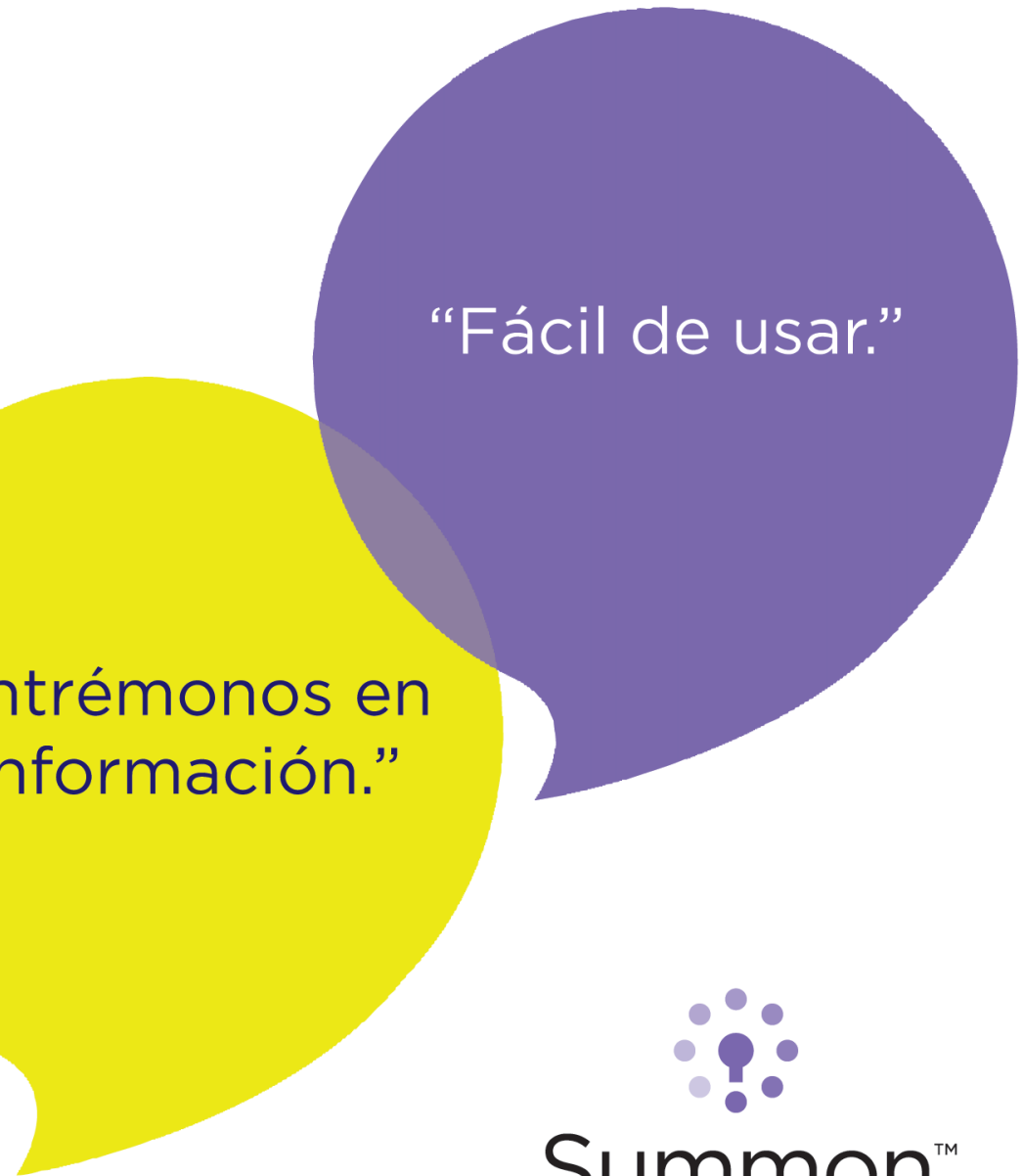